\title{
Signal- or temperature-based approach for cryoballoon ablation of atrial fibrillation: still an unsolved issue
}

\author{
Giacomo Mugnai ${ }^{1}$ (1) $\cdot$ Carlo de Asmundis $^{1} \cdot$ Gian-Battista Chierchia $^{1}$
}

Received: 20 May 2020 / Accepted: 1 June 2020 / Published online: 11 June 2020

(C) Springer Science+Business Media, LLC, part of Springer Nature 2020

Real-time (RT) recordings of the pulmonary vein (PV) electrograms can be visualized during the cryoballoon ablation (CB; Arctic Front Advance ${ }^{\mathrm{TM}}$, Medtronic, USA) using a dedicated inner lumen mapping catheter (ILMC: Achieve, Medtronic, USA). This tool provides useful information regarding time to isolation and on the conduction properties of the left atrium-pulmonary vein (LA-PV) junction [1]. Currently, there is a great variability in ablation protocols and dosing strategies among EP Centres. Two main approaches are today most frequently used during $\mathrm{CB}$ ablation: (1) a signal-guided approach in which the freezing duration and quantity are titrated by the "real-time" visualization of PV potentials and (2) a temperature-guided approach where the main target is to achieve a complete PV occlusion regardless of the presence of PV potentials and the ablation strategy is then guided by the temperatures attained during the freeze.

The latest technological modifications to the $\mathrm{CB}$ went in favor of the former approach promoting a higher rate of realtime visualization of PV EGMs. As a matter of fact, time to isolation has been shown to be one of the best predictor of persistency of electrical isolation following CB ablation $[2,3]$. Among these, the new Achieve included a new solid core providing improved rotational response, as compared with the previous version [4]. The greater flexibility and improved rotational response of the new device have provided a better positioning of the mapping catheter in the proximal portion of the PV resulting in improved visualization of electrical activity in the PV itself [4].

In addition, the third-generation $\mathrm{CB}$ was designed with a $40 \%$ shortened tip length compared with the former second-

Giacomo Mugnai

mugnai.giacomo@gmail.com

1 Heart Rhythm Management Center, UZ Brussel-VUB, Laarbeeklaan 101, 1090 Brussels, Belgium generation $\mathrm{CB}$ device $[5,6]$. Indeed, due to a more proximal positioning of the inner lumen mapping catheter, more RT recordings could be documented [5, 6]. However, due to certain technical issues, the third-generation $\mathrm{CB}$ was quickly removed from the market. In 2018, the fourth-generation CB became widely available bringing enhanced catheter maneuverability and ergonomics while still maintaining the shorter distal nose tip [7-9]. The fourth-generation CB seems to have visualization rates of PVs around $78 \%$, cumulative for all veins [7]; however, it is still controversial if this novel generation significantly increases the rate of PV potentials' visualization compared with the second generation $[8,9]$.

Despite the considerations mentioned above, real-time recordings might not be appreciated in up to $25 \%$ of veins [8]. On the other hand, the temperature-guided approach has the advantage of being a purely anatomical intervention entailing a considerable simplification and standardization of the procedure. This might hypothetically result in safer, shorter, and more reproducible procedural times.

A recent study sought to evaluate a temperature-guided approach based on the attainment of the specific parameter of $-40{ }^{\circ} \mathrm{C}$ within the first $60 \mathrm{~s}$ during $\mathrm{CB}$ applications, without the use of ILMC, compared with the conventional "electrogram-guided" approach [10]. A total of 52 patients were enrolled for each group (wire group vs ILMC group) and compared using the propensity score match analysis; over a mean follow-up period of $12.4 \pm 3.0$ months, the freedom from AF without antiarrhythmic drugs did not significantly differ between both groups. Of note, electrical isolation could be obtained in $99 \%$ of all PVs in the wire group, confirming the effectiveness of the temperature-guided approach. Although procedural times were not significantly different between the 2 groups, the fluoroscopy time was significantly reduced in the "wire group" [10]. In a previous article by Fürnkranz et al. [11], a nadir temperature of $<-51{ }^{\circ} \mathrm{C}$ proved to be a strong independent predictor of permanency of PV isolation. These findings were further confirmed by Scala et al. [12] in a recent publication. 
In conclusion, it is still controversial if a signal-guided approach during $\mathrm{CB}$ ablation should be preferred to a temperature-based approach. Larger, randomized trials of comparison are needed to determine if $\mathrm{CB}$ ablation can be performed by the sole guidance of complete PV occlusion and achieving a temperature cut-off in time without the need of verification of $\mathrm{PV}$ isolation. If this would prove true, temperature-guided $\mathrm{CB}$ ablation would further simplify the ablation procedure and make it more cost-effective.

\section{Compliance with ethical standards}

Conflict of interest $\mathrm{GBC}$ and $\mathrm{CdA}$ receive compensation for teaching purposes and proctoring from AF solutions, Medtronic . CdA is consultant for Daiichi Sankyo. GM received an educational grant from Medtronic for Postgraduate in Cardiac Electrophysiology and Pacing academic course.

\section{References}

1. Chierchia GB, de Asmundis C, Namdar M, Westra S, Kuniss M, Sarkozy A, et al. Pulmonary vein isolation during cryoballoon ablation using the novel Achieve inner lumen mapping catheter: a feasibility study. Europace. 2012;14(7):962-7.

2. Aryana A, Mugnai G, Singh SM, Pujara DK, de Asmundis C, Singh SK, et al. Procedural and biophysical indicators of durable pulmonary vein isolation during cryoballoon ablation of atrial fibrillation. Heart Rhythm. 2016;13(2):424-32.

3. Ciconte G, Mugnai G, Sieira J, Velagic V, Saitoh Y, Irfan G, et al. On the quest for the best freeze: predictors of late pulmonary vein reconnections after second-generation cryoballoon ablation. Circ Arrhythm Electrophysiol. 2015;8(6):1359-65.

4. Mugnai G, Manfrin M, de Asmundis C, Stroker E, Longobardi M, Rauhe $\mathrm{W}$, et al. The assessment of pulmonary vein potentials using the new achieve advance during cryoballoon ablation of atrial fibrillation. Indian Pacing Electrophysiol J. 2019;19(6):211-5.

5. Chierchia GB, Mugnai G, Stroker E, Velagic V, Hunuk B, Moran $\mathrm{D}$, et al. Incidence of real-time recordings of pulmonary vein potentials using the third-generation short-tip cryoballoon. Europace. 2016;18(8):1158-63.

6. Mugnai G, de Asmundis C, Hunuk B, Stroker E, Moran D, Hacioglu E, et al. Improved visualisation of real-time recordings during third generation cryoballoon ablation: a comparison between the novel short-tip and the second generation device. J Interv Card Electrophysiol. 2016;46(3):307-14.

7. Mathew S, Rottner L, Warneke L, Maurer T, Lemes C, Hashiguchi $\mathrm{N}$, et al. Initial experience and procedural efficacy of pulmonary vein isolation using the fourth-generation cryoballoon - a step forward? Acta Cardiol. 2019:1-6.

8. Moltrasio M, Sicuso R, Fassini GM, Riva SI, Tundo F, Dello Russo A, et al. Acute outcome after a single cryoballoon ablation: comparison between arctic front advance and arctic front advance PRO. Pacing Clin Electrophysiol. 2019;42(7):890-6.

9. Rottner L, Mathew S, Reissmann B, Warneke L, Martin I, Lemes $\mathrm{C}$, et al. Feasibility, safety, and acute efficacy of the fourthgeneration cryoballoon for ablation of atrial fibrillation: another step forward? Clin Cardiol. 2020;43(4):394-400.

10. Iacopino S, Mugnai G, Takarada K, Paparella G, Stroker E, De Regibus V, et al. Second-generation cryoballoon ablation without the use of real-time recordings: a novel strategy based on a temperature-guided approach to ablation. Heart Rhythm. 2017;14(3):322-8.

11. Furnkranz A, Koster I, Chun KR, Metzner A, Mathew S, Konstantinidou M, et al. Cryoballoon temperature predicts acute pulmonary vein isolation. Heart Rhythm. 2011;8(6):821-5.

12. Scala O, Borio G, Paparella G, Varnavas V, Stroker E, Guimaraes Osorio T, et al. Predictors of durable electrical isolation in the setting of second-generation cryoballoon ablation: a comparison between left superior, left inferior, right superior, and right inferior pulmonary veins. J Cardiovasc Electrophysiol. 2020;31(1):128-36.

Publisher's note Springer Nature remains neutral with regard to jurisdictional claims in published maps and institutional affiliations. 